\title{
A Novel Massage Therapy Technique for Management of Chronic Cervical Pain: A Case Series
}

William R. Thompson, DPT, PhD, ${ }^{1,4 *}$ Ronald Carter, LMT, NCBTMB, CPT, ${ }^{2,3,4 *}$ Benjamin Rohe, MS, ${ }^{1,3,4}$ Randall L. Duncan, PhD, ${ }^{1,3,4}$ Carlton R. Cooper, $\mathrm{PhD}^{1,3,4}$

\begin{abstract}
${ }^{1}$ Department of Biological Sciences, University of Delaware, Newark, ${ }^{2}$ The Massage Center, Wilmington, ${ }^{3}$ Center for Translational Cancer Research, Newark, ${ }^{4}$ Delaware Rehabilitation Institute, Newark, DE, USA. *Authors provided equal contribution to this work.
\end{abstract}

Background: Neck pain is a generalized condition resulting from a complex etiology with presentation of a wide variety of symptoms. Neck pain is most often accompanied by decreased range of motion (ROM), muscle and joint stiffness, and limitations in functional capabilities. This condition may result in significant personal and societal burden.

Purpose: We evaluated the effectiveness of a novel massage therapy intervention by following the treatment regimen and outcomes of two patients experiencing chronic neck pain.

Participants: Two patients (46 and 53 years old) experienced chronic ( $>5$ years) neck pain. Both patients reported pain, limited ROM, and muscle and joint stiffness. Additionally, the first patient reported a lack of sleep, and both patients stated their pain interfered with their quality of life and activities of daily living.

Intervention: Patients received the Integrative Muscular Movement Technique (IMMT) intervention approximately twice a week for a total of eight treatments, each approximately 20 minutes in duration.

Results: Both patients experienced a reduction in pain and an increase in cervical ROM in flexion, extension, rotation, and sidebending. The first patient also reported an increased ability to sleep. Both patients reported an increased ability to perform activities of daily living, including work-related responsibilities.

Conclusions: For the two patients included in this report, therapist observations and patient reports indicate that inclusion of the IMMT treatment in a treatment regimen for chronic neck pain may lead to decreased pain and increased cervical ROM. These positive effects of the IMMT intervention may have a role in enhancing functional outcomes of these patients.

KEYWORDS: Neck pain, massage therapy technique, chronic pain

\section{INTRODUCTION}

Neck pain is a frequent condition often resulting in significant individual and societal burden due to substantial health care costs and functional decline. ${ }^{(1)}$ Health care expenditures among individuals with spine-associated injuries have been estimated to exceed $\$ 90$ billion in $1998 .^{(2)}$ The average individual with spine pain incurred $60 \%$ higher health care costs than individuals without spinal pain $(\$ 3,498$ versus $\$ 2,178) .{ }^{(3)}$ Approximately $30 \%-50 \%$ of adults have neck pain in any given year, and about $50 \%-85 \%$ of individuals reporting neck pain do not return to their preinjury baseline with complete resolution of symptoms. ${ }^{(4)}$ Additionally, many individuals develop chronic pain that interferes with their activities of daily living (ADL). ${ }^{(5)}$ The prevalence of activity-impairing, chronic neck pain is estimated to range from $2.2 \%$ to $4.5 \%$ in the general population. ${ }^{(4,6,7)}$ While chronic neck pain alone may limit an individual's ability to engage in functional activities, there are numerous comorbidities associated with this condition. A large percentage $(87.1 \%)$ of people with chronic spinal pain reported at least one comorbid condition. The most common comorbidities were other chronic pain conditions $(68.6 \%)$ and cognitive disorders $(35.0 \%) .{ }^{(8)}$

Patients with chronic neck pain often present with a complex etiology. The complexity of neck pain may be attributed to confounding conditions such as joint hypomobility, often the result of the original trauma. This decreased cervical motion can lead to muscular trigger points that cause further pain and decreased range of motion (ROM), thus creating a vicious cycle of pain leading to decreased ROM, leading to further pain. ${ }^{(9)}$ Many individuals with chronic neck pain do not respond to typical courses of pain medication, and surgical approaches remain controversial due to mixed outcomes. ${ }^{(10)}$ Typical symptoms can range from headaches to limited ROM, radiculopathy, and generalized pain, all of which may lead to diminished quality of life and often loss of time at work. ${ }^{(7)}$ Additionally, studies demonstrate that $10 \%-22 \%$ of 
participants with chronic neck pain develop chronic widespread pain or fibromyalgia. ${ }^{(11-13)}$ These debilitating symptoms, combined with an increased likelihood of further exacerbation, emphasize the need for appropriate care and management of this pathology.

While treatments such as botulinum toxin injections, ${ }^{(14)}$ medial branch-block injections, radiofrequency neurotomy, ${ }^{(15)}$ and prescription narcotics ${ }^{(10)}$ have been used to address chronic neck pain, these treatments have limitations and sometimes serious adverse effects. ${ }^{(16)}$ Notably, opioid-derived medication use such as oxycodone, hydrocodone/acetaminophen, and morphine have several adverse effects including nausea, vomiting, dizziness, headaches, constipation, somnolence, and pruritus. These adverse effects were the primary cause of $30 \%-40 \%$ rates of attrition in studies involving these medications. ${ }^{(17-19)}$ These high rates of adverse effects make conservative treatments, such as massage therapy, a viable and attractive option for the reduction and management of chronic neck pain.

The most appropriate methods to treat chronic neck pain remain debatable. While several studies indicate that conservative treatments, including massage therapy, may be effective in reducing pain and improving outcomes for persons with chronic neck pain, ${ }^{(20,21)}$ the Cochrane review on massage for mechanical neck disorders was cautious in drawing definitive conclusions. It stated that no recommendations for practice could be made with regard to the effectiveness of massage for neck pain, primarily because the overall quality of the studies available for review was judged to be poor. ${ }^{(21)}$ The Cochrane review demonstrates the importance of ongoing investigations into the effectiveness of massage therapy.

Here we present the cases of two patients who were experiencing chronic neck pain for 5 years or more. A novel therapeutic intervention termed Integrative Muscular Movement Technique (IMMT), which will be described here for the first time, was applied. The aim of this treatment approach was to reduce the severity of the patient's symptoms, which included pain and limited ROM, with the end goal to help each patient return to their desired level of function.

\section{METHODS}

\section{Client Profile}

Patient A was a 46-year-old woman who presented with migraine headaches lasting most of the day and occurring 9-13 times per month. Her headaches were accompanied by cervical and shoulder stiffness and muscle spasms. Additionally, she reported a lack of sleep, which often limited her ability to fully engage her responsibilities at work and home. The patient's symptoms, including her insomnia, began approximately 5 years earlier when she was involved in a motor vehicle accident. She attributed the cervical pain, headaches, and inability to sleep to the injuries sustained in the accident. Prior to the onset of her symptoms, the patient was independent in all ADLs and participated in a variety of activities with her family without any functional limitations. She held a full-time job as a certified occupational therapy assistant and did not go on disability or lose any time at work due to her injury. The patient had never previously experienced massage or any other complementary therapy.

Patient B was a 53-year-old woman who presented with limited cervical ROM and chronic pain that had been persistent for the past 5 years. She attributed this pain to extended periods of poor posture and overuse necessitated by her job as a dental hygienist. The pain and limited ROM led to difficulty turning her head during activities such as driving and were severely aggravated by her work responsibilities. Prior to her pain, this patient experienced no functional limitations in any of her ADLs, including a variety of activities that she enjoyed with her family and friends, and had been able to interact with dental patients and desk work for more than 6 hours a day. During work hours her duties led her to adopt a forward-flexed posture most of the work day. Prior to this treatment, the patient had never experienced massage or any other complementary therapy.

\section{Therapist Profile}

The same therapist, a man with over 11 years of experience in massage therapy, treated both patients. The therapist obtained licensure in deep muscle therapy with a 600-hour course in the subject. Additionally, he had completed a 200-hour course in shiatsu massage and had owned his own massage therapy practice for 6 years.

\section{Intervention}

The IMMT is a novel massage intervention incorporating principles of myofascial release with active isolated stretching. Each patient received the IMMT treatment approximately twice a week for 4 weeks, for a total of eight treatments. Each treatment lasted approximately 20 minutes. The treatment duration and frequency was predetermined based on the perceived ability of each patient to tolerate the intervention. Prior to each treatment each patient's pain levels were recorded using a $0-10$ verbal pain scale, where $0=$ no pain and $10=$ the most extreme pain. Additionally, ROM measurements (cervical flexion, extension, sidebending, and rotation) were assessed for each patient before and after each treatment. Flexion and extension were measured by aligning the axis of the goniometer with the center of the ear; the stationary arm was aligned vertically with the head and the moving arm followed the tip of the patient's 
nose. Cervical sidebending was measured with the axis of the goniometer aligned with the seventh cervical vertebra; the stationary arm was aligned vertically with the external occipital protuberance of the skull and the moving arm followed the head during sidebending. Rotation was measured with the axis of the goniometer at the center of the top of the skull; the stationary arm was aligned with the patient's nose and the moving arm followed the nose during the rotation movement.

The goal of the novel IMMT was to take advantage of compressive movements to help release myofascial adhesions (formation of excess fibrous connective tissue) while also incorporating short, controlled stretches with intervals of relaxation to override the tendency of the muscle to contract when stretched tightly. This approach focuses on four major muscle groups of the cervical region: the sternocleidomastoid (SCM), the scalene muscle group, the splenius muscle group, and the suboccipital muscle group. The potential benefits (decreased pain, reduced stiffness, and increased ROM) and risks (temporary discomfort and redness, and skin irritation) of the IMMT were described in detail, and informed consent was obtained from both patients prior to the initiation of treatment. The massage technique used for the patients described in this case series began with the patient lying supine. The treatment began with 5-10 strokes of effleurage using Swedish effleurage massage oil (TheraPro, Norfolk, VA, USA). Following effleurage, each treatment began by focusing on the SCM muscle and then continued to each subsequent muscle in the order mentioned above.

To begin the IMMT procedure the therapist placed one hand at the base of the muscle (eg, clavicular attachment of the SCM), using a firm grip to "pin" the muscle. The therapist's other hand was placed on the patient's head (Figure 1). The muscle would then be placed in a compressed position (eg, cervical flexion for the SCM) by movement of the head via passive motion guided by the therapist. This compressed position was held for 2-3 seconds followed by movement of the head into a stretched position (eg, cervical extension for the SCM), which was held for 2-3 seconds. Following the compression and stretching phases, the muscle was returned to a neutral position, and the hand gripping the muscle then was moved to the middle of the muscle belly to once again pin the muscle in place while the compression and stretching actions were repeated once more. The hand used to pin the muscle was moved up the entire muscle each time, and at each position the muscle was moved into the compression and stretching positions (Figure 1). For each muscle treated, the muscle would be moved in all of its possible movements while the muscle would be pinned in each position as mentioned above. For example, the SCM muscle would be compressed/ stretched by moving in to cervical flexion/extension as the hand was worked up the muscle belly as
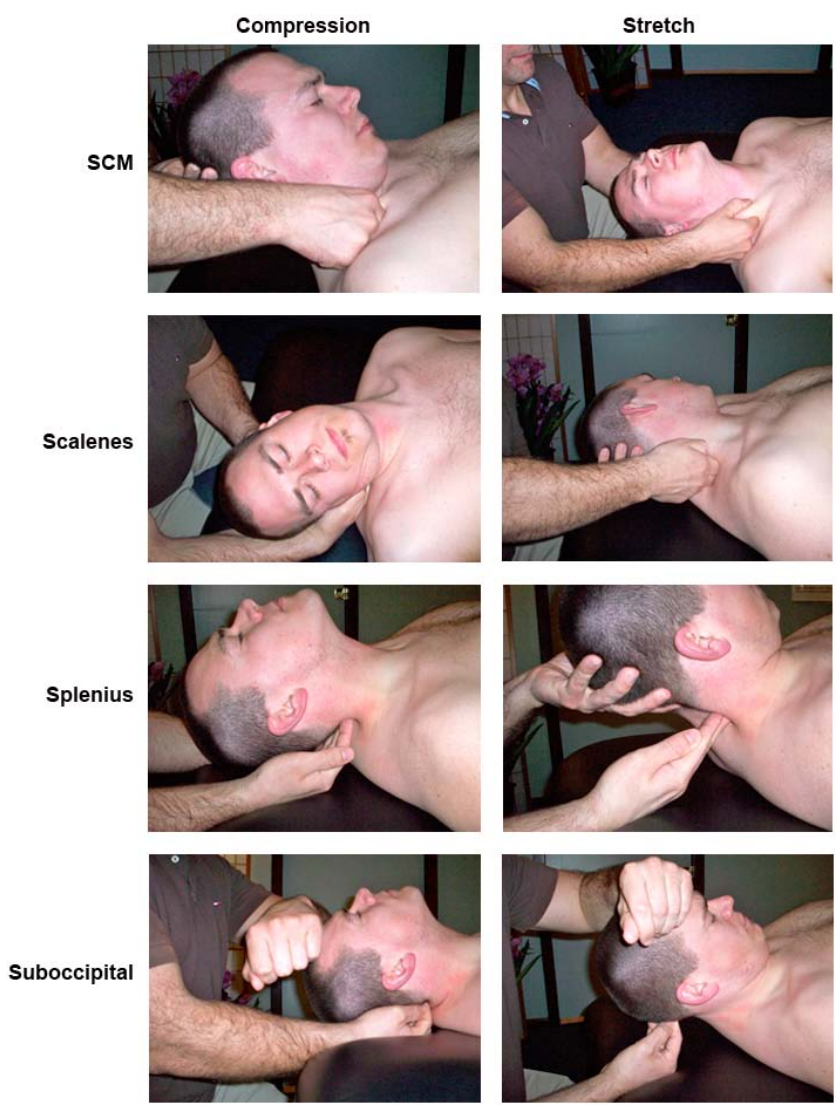

FIGURE 1. Integrative Muscular Movement Technique (IMMT) therapist and patient positioning. The patient was positioned in supine for the duration of the treatment. The muscle being addressed was "pinned" by the therapist's hand (therapist's right hand in each panel). The head was then moved in a position of compression or stretch according to the muscle being treated as detailed for the sternocleidomastoid (SCM), scalene muscle group, splenius muscle group, and suboccipital muscle group. The therapist's left hand was used to passively move the patient's head in the appropriate cervical position. The individual pictured was not a patient in the current study.

described above. This would then be followed by contralateral rotation/ipsilateral rotation and then ipsilateral sidebending/contralateral sidebending. Figure 2 details the compression/stretching patterns for each muscle treated.

\section{RESULTS}

Over the course of eight treatments (over a 4- to 6-week interval) patient A experienced an overall reduction in pain from a rating of 6 (out of 10) to 2 and had a self-reported increased ability to sleep. Patient B saw a decrease in pain from 2 to 1 out of 10 (Figure 3). There was an initial increase in pain levels between the first and third treatment sessions (Figure 3). This increase is most likely due to an acute reaction to the treatment. The IMMT introduces an 


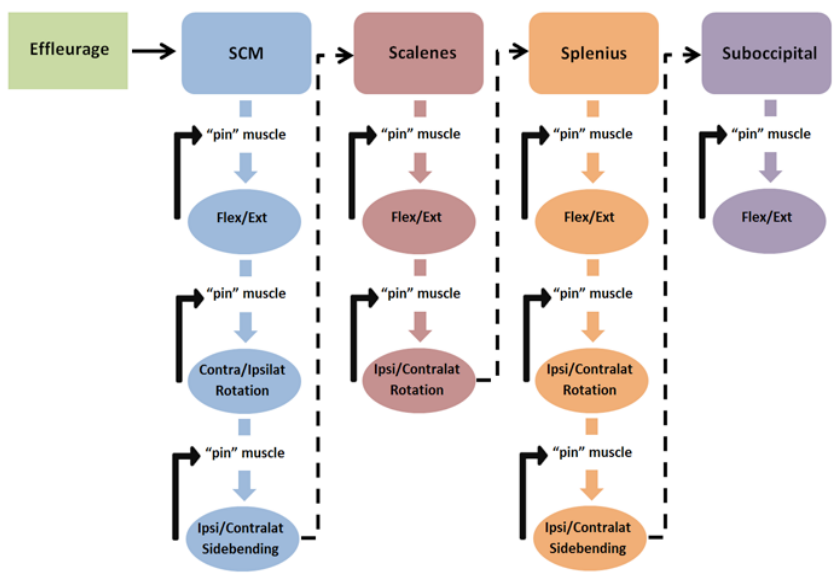

FIGURE 2. Sequence of progression of Integrative Muscular Movement Technique (IMMT). The IMMT begins with 5-10 strokes of effleurage to warm the muscles. The sternocleidomastoid (SCM) muscle then is addressed. The therapist first uses the right hand to "pin" the muscle at its base (clavicular attachment for SCM). The head then is moved into flexion to compress the SCM and is held for 2-3 seconds followed by movement into extension for 2-3 seconds to stretch the muscle. The muscle then is placed in a neutral position, the hand pinning the muscle is moved to a position higher along the muscle belly, and the flexion (Flex)/extension (Ext) movement is repeated (denoted by solid black arrow). This sequence is repeated along the entire length of the muscle belly (typically 2-3 repetitions per muscle). The therapist then remains on the SCM and begins the same procedure except moving the head into contralateral (contralat) or ipsilateral (ipsilat) rotation (progression to the next movement denoted by colored downward arrow). The muscle is initially pinned at the base as before and the procedure is repeated along the length of the muscle. This procedure is repeated for each movement as described above for the SCM; then the therapist moves to the scalene muscle group completing the procedure for the involved motions, followed by the splenius muscle group, and lastly the suboccipital muscle group (progression between muscles denoted by dotted arrow). The entire treatment lasts approximately 20 minutes.

aggressive stretch likely to induce discomfort during the initial phase of the treatment. Despite the initial rise in pain, both patients experienced an overall reduction in their self-reported pain levels over the eight sessions. Additionally, both patients reported that the reduction of pain associated with the treatment resulted in an increased ability to perform functions related to their ADLs, including work, driving, and home-related responsibilities.

In addition to improvements in overall pain levels, the IMMT treatment resulted in enhanced ROM in cervical flexion, extension, sidebending, and rotation compared with each patient's pretreatment measurements. Both patients experienced an improvement in ROM for all motions in the posttreatment measurement compared with the pretreatment ROM for all eight treatments. Additionally, overall ROM in all cervical movements increased in both patients at the posttreatment measurement on the final day of treatment compared with the pretreatment ROM for the first treatment. For patient A, the final ROM

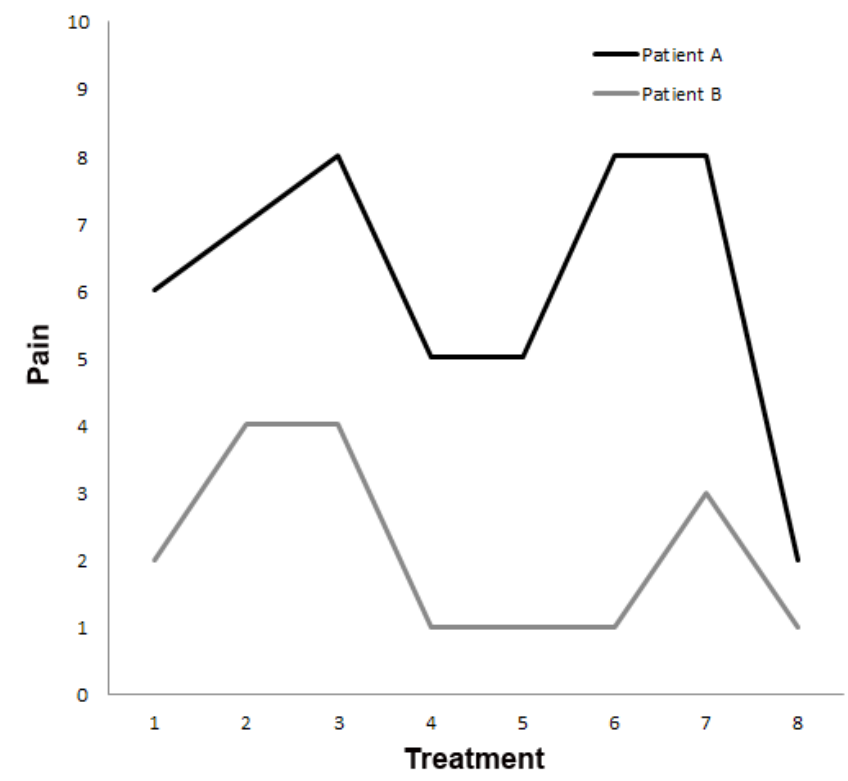

FIGURE 3. Pain ratings as reported by each patient. Pain ratings were obtained before each treatment session and were reported on a scale of $0-10$ with $0=$ no pain and $10=$ worst possible pain. Patient A: decrease in pain from 6 to 2; Patient B: decrease in pain from 2 to 1 over the course of 8 treatments. For both patients, pain increased initially and peaked at treatment 3. This increase may be attributed to discomfort experienced by the initiation of the massage technique.

measurement following the last treatment increased over the pretreatment measurement of the first treatment by $33^{\circ}(62 \%)$ in cervical flexion, $34^{\circ}(55 \%)$ in extension, $16^{\circ}(80 \%)$ in right sidebending, $19^{\circ}(100 \%)$ in left sidebending, $14^{\circ}(21 \%)$ in right rotation, and $19^{\circ}(32 \%)$ in left rotation (Table 1). Patient B's ROM had improved similarly on the last day of treatment of IMMT compared with the first day. Patient B showed an increase of $45^{\circ}(100 \%)$ in cervical flexion, $20^{\circ}$ $(27 \%)$ in extension, $16^{\circ}(46 \%)$ in right sidebending, $16^{\circ}(46 \%)$ in left sidebending, $25^{\circ}(33 \%)$ in right rotation, and $23^{\circ}(30 \%)$ in left rotation (Table 2$)$.

Overall, both patients reported being satisfied with the results achieved with the IMMT treatment. While the trajectory of pain for both patients shows an increase in pain after one to two treatments followed by another increase in pain just prior to the final treatment, an overall reduction in pain and a consistent increase in ROM in all tested movements over the course of the eight treatments were observed in both patients. Following the completion of all treatments, both patients reported an increased ability to participate in work and home-related activities.

\section{DISCUSSION}

The goal of this case series was to describe the novel IMMT massage intervention and to detail 
TABle 1. Patient A's Range of Motion Measurements ${ }^{\mathrm{a}}$

\begin{tabular}{|c|c|c|c|c|c|c|c|c|c|c|c|c|}
\hline & \multicolumn{2}{|c|}{ Flexion } & \multicolumn{2}{|c|}{ Extension } & \multicolumn{2}{|c|}{ Right sidebending } & \multicolumn{2}{|c|}{ Left sidebending } & \multicolumn{2}{|c|}{ Right rotation } & \multicolumn{2}{|c|}{ Left rotation } \\
\hline & Range & $P I$ & Range & $P I$ & Range & $P I$ & Range & $P I$ & Range & $P I$ & Range & $P I$ \\
\hline Pre-tx day 1 & $53^{\circ}$ & - & $62^{\circ}$ & - & $20^{\circ}$ & - & $19^{\circ}$ & - & $68^{\circ}$ & - & $60^{\circ}$ & - \\
\hline Post-tx day 1 & $63^{\circ}$ & $19 \%$ & $83^{\circ}$ & $34 \%$ & $36^{\circ}$ & $80 \%$ & $33^{\circ}$ & $74 \%$ & $77^{\circ}$ & $13 \%$ & $73^{\circ}$ & $22 \%$ \\
\hline Pre-tx day 8 & $77^{\circ}$ & $45 \%$ & $90^{\circ}$ & $45 \%$ & $33^{\circ}$ & $65 \%$ & $35^{\circ}$ & $84 \%$ & $82^{\circ}$ & $21 \%$ & $78^{\circ}$ & $30 \%$ \\
\hline Post-tx day 8 & $86^{\circ}$ & $62 \%$ & $96^{\circ}$ & $55 \%$ & $36^{\circ}$ & $80 \%$ & $38^{\circ}$ & $100 \%$ & $82^{\circ}$ & $21 \%$ & $79^{\circ}$ & $32 \%$ \\
\hline
\end{tabular}

${ }^{a}$ Measured in degrees and percentage increase (PI) compared with pretreatment (Pre-tx) day 1 . Post-tx $=$ posttreatment.

TABle 2. Patient B's Range of Motion Measurements ${ }^{\mathrm{a}}$

\begin{tabular}{|c|c|c|c|c|c|c|c|c|c|c|c|c|}
\hline & \multicolumn{2}{|c|}{ Flexion } & \multicolumn{2}{|c|}{ Extension } & \multicolumn{2}{|c|}{ Right sidebending } & \multicolumn{2}{|c|}{ Left sidebending } & \multicolumn{2}{|c|}{ Right rotation } & \multicolumn{2}{|c|}{ Left rotation } \\
\hline & Range & $P I$ & Range & $P I$ & Range & $P I$ & Range & $P I$ & Range & $P I$ & Range & $P I$ \\
\hline Pre-tx day 1 & $45^{\circ}$ & - & $74^{\circ}$ & - & $35^{\circ}$ & - & $35^{\circ}$ & - & $75^{\circ}$ & - & $76^{\circ}$ & - \\
\hline Post-tx day 1 & $71^{\circ}$ & $58 \%$ & $89^{\circ}$ & $20 \%$ & $35^{\circ}$ & $0 \%$ & $44^{\circ}$ & $26 \%$ & $96^{\circ}$ & $28 \%$ & $82^{\circ}$ & $8 \%$ \\
\hline Pre-tx day 8 & $83^{\circ}$ & $84 \%$ & $88^{\circ}$ & $19 \%$ & $45^{\circ}$ & $29 \%$ & $48^{\circ}$ & $37 \%$ & $93^{\circ}$ & $24 \%$ & $89^{\circ}$ & $17 \%$ \\
\hline Post-tx day 8 & $90^{\circ}$ & $100 \%$ & $94^{\circ}$ & $27 \%$ & $51^{\circ}$ & $46 \%$ & $51^{\circ}$ & $46 \%$ & $100^{\circ}$ & $33 \%$ & $99^{\circ}$ & $30 \%$ \\
\hline
\end{tabular}

a Measured in degrees and percentage increase (PI) compared with pretreatment (Pre-tx) day 1. Post-tx $=$ posttreatment.

the treatment course of two patients receiving this intervention following chronic neck pain. This technique was designed to incorporate principles of myofascial release, reduce myofascial adhesions, and combine stretching and compression components to the muscle to increase ROM and reduce the effects of trigger points.

Patients experiencing chronic neck pain are often presented with a wide variety of treatment options from physicians, surgeons, physical therapists, chiropractors, and massage therapists. Given the limitations of some treatments and the frequently reported adverse effects of certain medications and invasive procedures, a multidisciplinary approach incorporating appropriate conservative treatments is often indicated. The IMMT method may serve as an additional tool for massage therapists and rehabilitation specialists to treat patients with chronic neck pain.

Both patients in this case series experienced a reduction in their overall pain over the course of eight treatments. Additional studies are underway that include the implementation of a longer treatment course, which may influence the resolution of pain symptoms. Additionally, both patients reported the ability to participate more fully in their ADLs; however, this study did not quantitatively assess the functional outcomes of each patient on a longitudinal scale. The initiation of treatment for both of these patients was 5 years or more after the initial injury. While the IMMT would most likely not be indicated for patients after an acute injury, due to the aggressive nature of the muscle stretch, patients who are in the subacute phase following cervical injuries of muscular origin may benefit from this technique. Additional studies are ongoing to elucidate the appropriate treatment course, including the most effective time after injury to introduce this intervention.

Because this case series details the treatment progression of only two patients it is important to recognize that the approach described here is not intended to be a generalized treatment for all patients with neck pain. Neck pain often results from a complex etiology and caution must be taken to ensure that conditions such as fracture or nerve injury have not occurred prior to the initiation of any massage intervention. The IMMT treatment approach is intended to augment additional interventions with the oversight of a physician. Additionally, this technique may be beneficial when more conventional treatment approaches have been unsuccessful.

The most common complaint from both patients receiving this treatment was that the compression and stretch component of the intervention was often uncomfortable and sometimes painful. The trajectory of pain ratings from each patient (Figure 3) rose initially early in the treatment course with an eventual decline in pain followed by another increase. Ultimately, both patients reported an overall reduction of pain at the end of eight treatment sessions; however, the initial discomfort experienced with this massage regimen may be too uncomfortable for some patients, leading to decreased treatment compliance. The discomfort reported by each patient resulted in some muscle guarding during the treatment. To maintain 
a consistent treatment course over the eight sessions the treatment was not augmented because of the discomfort. In light of the feedback of pain with the initiation of the IMMT we are now exploring a less aggressive form of the technique to enhance patient comfort and compliance. To promote relaxation and to prevent additional muscle guarding, we recommend implementing rest periods and providing a longer period of effleurage prior to the stretching and compression component of the IMMT. Adding effleurage to the end of the treatment may also help the patient relax and increase treatment compliance.

We have several other recommendations when considering implementing the IMMT method for chronic neck pain. It is essential that the patient and the therapist have an open dialogue concerning the expectations of the treatment progression. The patient must understand that there may be adverse effects, such as temporary discomfort, related to the treatment. While some discomfort may accompany each treatment, the lasting effect of the intervention will most likely outweigh this. Patient feedback is encouraged before, during, and after each treatment session, in order to determine which components of the intervention are most helpful. Additionally, patients can benefit from instruction on proper posture to help prevent undue stress or strain on their cervical spine during work, driving, or activities at home.

Both patients described in this case series reported that they were satisfied with the overall progression of the treatment and the reduction of their pain. Most notably, they were pleased with their increased ability to participate in activities in which they were previously limited due to their pain and limited ROM. Patient A also commented on the enhanced ability to sleep, which she attributed to a reduction in her neck pain.

Future studies with a larger patient population are underway. Additionally, for more accurate assessments, additional questionnaires should be employed. These may include self-reports, functional assessments such as The Functional Status Questionnaire, ${ }^{(22)}$ physiological measures such as the McGill Pain Questionnaire, ${ }^{(23)}$ or indicators of functional decline such as the Neck Disability Index. ${ }^{(24)}$ These tools may more accurately assess the results of the treatment plan, assisting the therapist and patient in monitoring progress.

In this case series we have detailed the progression of two patients treated with a novel massage intervention called IMMT. Both patients, who had chronic neck pain, experienced reduced pain, increased ROM, and an increased ability to participate in regular daily activities after an eight-treatment course of IMMT. These positive effects indicate that this treatment may be beneficial as an additional option for patients with musculoskeletal cervical pathologies. This treatment protocol and the related guidelines discussed here can contribute to further studies of patients with chronic neck pain seeking alternative therapy options.

\section{ACKNOWLEDGMENTS}

Funding support: This study was supported by Foundation for Physical Therapy Florence Kendall Scholarship, Foundation for Physical Therapy Promotion of Doctoral Studies II training fellowship, and the American Physical Therapy Association Section on Geriatrics Adopt-A-Doc Scholarship to WRT and The Center for Translational Cancer Research to BR and CRC.

\section{CONFLICT OF INTEREST NOTIFICATION}

All authors have no conflicts of interest.

\section{COPYRIGHT}

Published under the CreativeCommons AttributionNonCommercial-NoDerivs 3.0 License.

\section{REFERENCES}

1. Carroll LJ, Cassidy JD, Peloso PM, et al. Methods for the best evidence synthesis on neck pain and its associated disorders: the Bone and Joint Decade 2000-2010 Task Force on Neck Pain and Its Associated Disorders. Spine (Phila Pa 1976). 2008;33(4 Suppl):S33-S38.

2. Luo X, Pietrobon R, Sun SX, et al. Estimates and patterns of direct health care expenditures among individuals with back pain in the United States. Spine (Phila Pa 1976). 2004;29(1):79-86.

3. Bell GK, Kidd D, North RB. Cost-effectiveness analysis of spinal cord stimulation in treatment of failed back surgery syndrome. J Pain Symptom Manage. 1997;13(5):286-295.

4. Haldeman S, Carroll L, Cassidy JD, et al. The Bone and Joint Decade 2000-2010 Task Force on Neck Pain and Its Associated Disorders: executive summary. Spine (Phila Pa 1976). 2008;33(4 Suppl):S5-S7.

5. Carroll LJ, Hogg-Johnson S, van der Velde G, et al. Course and prognostic factors for neck pain in the general population: results of the Bone and Joint Decade 2000-2010 Task Force on Neck Pain and Its Associated Disorders. Spine (Phila Pa 1976). 2008;33(4 Suppl):S75-S82.

6. Chiu TT, Leung AS. Neck pain in Hong Kong: a telephone survey on prevalence, consequences, and risk groups. Spine (Phila Pa 1976). 2006;31(16):E540-E544.

7. Goode AP, Freburger J, Carey T. Prevalence, practice patterns, and evidence for chronic neck pain. Arthritis Care Res (Hoboken). 2010;62(11):1594-1601.

8. Von Korff M, Crane P, Lane M, et al. Chronic spinal pain and physical-mental comorbidity in the United States: results from the national comorbidity survey replication. Pain. 2005;113(3):331-339.

9. Fernandez-de-Las-Penas C. Interaction between trigger points and joint hypomobility: a clinical perspective. J Man Manip Ther. 2009;17(2):74-77. 
10. Ma K, Jiang W, Zhou Q, et al. The efficacy of oxycodone for management of acute pain episodes in chronic neck pain patients. Int J Clin Pract. 2008;62(2):241-247.

11. Andersson HI. The course of non-malignant chronic pain: a 12-year follow-up of a cohort from the general population. Eur J Pain. 2004;8(1):47-53.

12. Buskila D, Neumann L, Vaisberg G, et al. Increased rates of fibromyalgia following cervical spine injury: a controlled study of 161 cases of traumatic injury. Arthritis Rheum. 1997;40(3):446-452.

13. Holm LW, Carroll LJ, Cassidy JD, et al. Widespread pain following whiplash-associated disorders: incidence, course, and risk factors. $J$ Rheumatol. 2007;34(1):193-200.

14. Langevin P, Lowcock $\mathrm{J}$, Weber $\mathrm{J}$, et al. Botulinum toxin intramuscular injections for neck pain: a systematic review and metaanalysis. J Rheumatol. 2011;38(2):203-214.

15. Schofferman J, Bogduk N, Slosar P. Chronic whiplash and whiplash-associated disorders: an evidence-based approach. $J$ Am Acad Orthop Surg. 2007;15(10):596-606.

16. Datta S, Lee M, Falco FJ, et al. Systematic assessment of diagnostic accuracy and therapeutic utility of lumbar facet joint interventions. Pain Physician. 2009;12(2):437-460.

17. Hale ME, Dvergsten C, Gimbel J. Efficacy and safety of oxymorphone extended release in chronic low back pain: results of a randomized, double-blind, placebo- and active-controlled phase III study. J Pain. 2005;6(1):21-28.

18. Hale ME, Ahdieh H, Ma T, et al. Efficacy and safety of OPANA ER (oxymorphone extended release) for relief of moderate to severe chronic low back pain in opioid-experienced patients: a 12-week, randomized, double-blind, placebo-controlled study. J Pain. 2007;8(2):175-184.
19. Katz N, Rauck R, Ahdieh H, et al. A 12-week, randomized, placebo-controlled trial assessing the safety and efficacy of oxymorphone extended release for opioid-naive patients with chronic low back pain. Curr Med Res Opin. 2007;23(1):117-128.

20. Sherman KJ, Cherkin DC, Hawkes RJ, et al. Randomized trial of therapeutic massage for chronic neck pain. Clin J Pain. 2009;25(3):233-238.

21. Kay TM, Gross A, Goldsmith C, et al. Exercises for mechanical neck disorders. Cochrane Database Syst Rev. 2005;(3):CD004250.

22. Jette AM, Davies AR, Cleary PD, et al. The Functional Status Questionnaire: reliability and validity when used in primary care. J Gen Intern Med. 1986;1(3):143-149.

23. Corson JA, Schneider MJ. The Dartmouth Pain Questionnaire: an adjunct to the McGill Pain Questionnaire. Pain 1984;19(1):59-69.

24. Vernon H, Mior S. The Neck Disability Index: a study of reliability and validity. J Manipulative Physiol Ther. 1991;14(7):409-415.

Corresponding author: William R. Thompson, DPT, PhD, Department of Biological Sciences, University of Delaware, 317 Wolf Hall, 105 The Green, Newark, DE 19716 USA

E-mail: wthomp@udel.edu 